\title{
Influence of Magnetic Fields and Biasing on the Plasma of a RF Driven Negative Ion Source
}

\author{
D. Wünderlich, R. Gutser, U. Fantz and the NNBI Team \\ Max Planck-Institut für Plasmaphysik, Boltzmannstr. 2, D-85748 Garching bei München
}

\begin{abstract}
The development of a RF driven negative-ion source currently in progress at IPP Garching has made remarkable progresses during the last years. Optimization of the source strongly depends on the understanding of the physics of generation, destruction and extraction of negative ions. Numerical model calculations can provide important information needed for the improvement of this understanding. Since negative ions are mainly produced on Cesium covered surfaces of the source, and have a survival length in the range of a few $\mathrm{cm}$, models for the region close to the plasma grid are of particular interest. The plasma in this region is affected by magnetic filter fields. Another important feature is biasing of the plasma grid which strongly influences the co-extracted electron current. The different effects are treated in two separate models. Their current status is presented: a particle-in-cell-code (PIC) is used to study the influence of filter fields and bias voltage on the plasma flow onto the plasma grid. The motion of the charged particles due to their own and externally applied electric field is simulated in a selfconsistent way. In the second code the survival probability of negative ions produced on the surface of the plasma grid is estimated using test-particles. The Monte-Carlo technique is applied for the treatment of inelastic and elastic collisions.
\end{abstract}

Keywords: Plasma simulation, Particle-in-cell, Particle orbit and trajectory, Monte Carlo methods.

PACS: 52.65.-y, 52.65.Rr, 52.65.Cc, 52.65.Pp

\section{INTRODUCTION}

For heating and current drive of future fusion devices neutral beam injection based on negative hydrogen ions is required. The ion source used for ITER should deliver 40 A of $\mathrm{D}^{-}$ions with a current density of $200 \mathrm{~A} / \mathrm{m}^{2}$ at a source pressure of $0.3 \mathrm{~Pa}$ and an electron to ion ratio $<1$ [1]. At IPP Garching RF driven ion sources are under development which has already fulfilled some of these requirements [2]. Figure 1 shows a schematic diagram of the so-called type 6/1 source.

The source consists of three parts: The driver where the RF is coupled to the plasma, the expansion region where the plasma expands into the source volume and the extraction region close to the extraction system. A magnetic filter between expansion region and extraction region removes hot electrons $\left(\mathrm{T}_{\mathrm{e}}>2 \mathrm{eV}\right)$ flowing into the direction of the extraction system. Removing these electrons is necessary to reduce the destruction of negative ions which occurs mainly by electron stripping and mutual neutralization with $\mathrm{H}^{+}$. While the probability for electron stripping decreases rapidly with decreasing electron temperature for $\mathrm{T}_{\mathrm{e}}<10 \mathrm{eV}$ [3], mutual neutralization is 


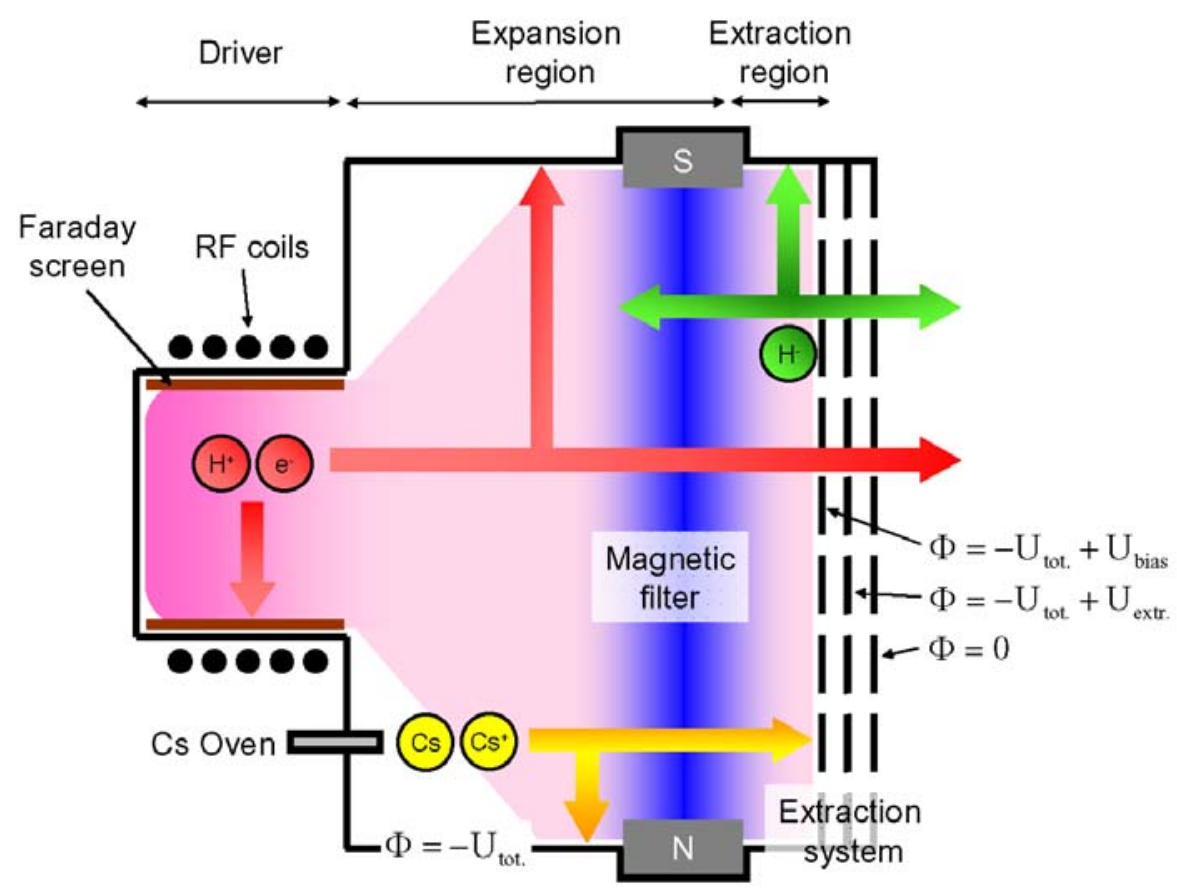

FIGURE 1. Schematic diagram of the type 6/1 RF ion source. Typical values for the voltages are: $\mathrm{U}_{\text {extr. }}=10 \mathrm{kV}, \mathrm{U}_{\text {tot. }}=20 \mathrm{kV}, \mathrm{U}_{\text {bias }}=20 \mathrm{~V}$

almost independent of the electron temperature. The rate coefficients for both processes are equal at $\mathrm{T}_{\mathrm{e}}=2 \mathrm{eV}$, thus the destruction of $\mathrm{H}^{-}$is minimized for $\mathrm{T}_{\mathrm{e}}<2 \mathrm{eV}$.

Negative ions are produced mainly by the surface effect [2]: Hydrogen particles $(\mathrm{H}$ or $\mathrm{H}^{+}$) pick up electrons from surfaces which have a low work function due to the coverage by a thin cesium film. The value of the work function depends on the thickness of the Cs film [4]. A Cesium oven with adjustable evaporation rate is connected to the back flange of the source body. Ions produced on the surfaces are accelerated towards the plasma volume by the sheath potential. Since at typical plasma parameters of the ion source $\left(\mathrm{T}_{\mathrm{e}}=3 \mathrm{eV}, \mathrm{n}_{\mathrm{e}}=5 \cdot 10^{17} \mathrm{~m}^{-3}\right.$ in the extraction region for 120 $\mathrm{kW}$ input power at $0.5 \mathrm{~Pa}$ ) the survival length of the negative hydrogen ions is in the range of a few $\mathrm{cm}$, only ions produced on the surface of the plasma grid are of importance for extraction. The geometry of the grid is of importance for the negative ion production rate: by introducing a plasma grid with chamfered edges the extracted ion current is increased [2].

The extraction system consists of three grids: the plasma grid, the extraction grid and the grounded grid. Rods made of CoSm are placed inside the extraction grid and create a magnetic field to prevent the co-extracted electrons from being fully accelerated.

Experimental data shows that the absolute extracted ion current as well as the ratio of ion current to electron current can easily be changed by varying the bias voltage of the plasma grid and the strength of the magnetic filter field $[2,5]$. Varying bias voltage and filter strength has collective effects on the plasma, i.e. the fluxes of the charged particles to the walls of the source are altered and thus the plasma potential is changed. This has an effect on the dynamics of the negative ions produced at the plasma grid 
surface. A complete understanding of the complex effects involved thereby in an ion source dominated by surface production is essential for the further optimization of the source. The aim of this paper is to present two numerical models which can improve the insight in the effects described above.

\section{SIMULATION MODEL}

For the sake of simplicity two different models are used: A Particle-in-cell (PIC) code and a test particle code. The PIC code is used to study the influence of biasing and the magnetic filter field on the plasma as a whole. The test particle code analyzes the influence of bias and filter field on the dynamics of negative ions produced on the plasma grid surface. Both codes will be described in detail in the following.

\section{Particle-In-Cell Code}

The motion of charged particles (electron, positive and negative hydrogen ions) is calculated by a 2d-3v Particle-in-cell (PIC) code in a self-consistent way. All particles are treated simultaneously. Since the number of particles in a plasma is typically very large, pseudo particles are introduced. Each pseudo particle represents a certain number of real particles. Calculations are based on a grid in time and space. The finite difference method is used, i.e. the space grid consists of quadratic cells. The size of time steps and grid cells is chosen to be small enough to resolve plasma oscillations $\left(\Delta \mathrm{t}<1 / \omega_{\mathrm{Pl}}, \Delta \mathrm{x}, \Delta \mathrm{y}<\lambda_{\mathrm{D}}\right)[6]$ where $\omega_{\mathrm{Pl}}$ is the plasma frequency and $\lambda_{\mathrm{D}}$ the Debye length. Thus for the chosen domain size the current version of the code $(60 \times 50$ grid cells) is restricted to low plasma densities $\left(\mathrm{n}_{\mathrm{e}} \approx 10^{15} \mathrm{~m}^{-3}\right.$, this is much lower than the density inside the experiment which is around $10^{17} \mathrm{~m}^{-3}$ ). Calculations for higher plasma densities would require remarkable more computational time. The geometry of the computational domain is shown in Fig. 2. The test line indicated in this figure will be used for illustrating the results of the code.

The code focuses on a small region close to the plasma grid. The dimension of the domain is $3.0 \mathrm{~cm}$ in $\mathrm{x}$ direction and $2.5 \mathrm{~cm}$ in y direction. Instead of using periodic boundary conditions for the upper and lower boundary walls are introduced since the flux of particles deflected inside the magnetic filter and hitting the walls is important for the formation of the plasma potential. Therefore upper and lower boundaries are set to a potential of $0 \mathrm{~V}$; the electrode and the right wall are set to the bias and extraction voltage respectively. The bias voltage is a parameter of the calculations. The shape of the extraction surface depends on the perveance $\Pi=I / U^{1.5}$. Since the particle fluxes in the model are low compared to the experiment, a remarkably smaller extraction voltage was chosen for the model than present in the experiment to obtain a similar perveance [7]: $50 \mathrm{~V}$ compared to roughly $10000 \mathrm{~V}$. At the left wall the derivation of the potential is fixed to zero since this wall represents the connection of the simulation domain to the plasma volume.

As initial condition the domain contains no particles. For each time step the following procedure is performed: 


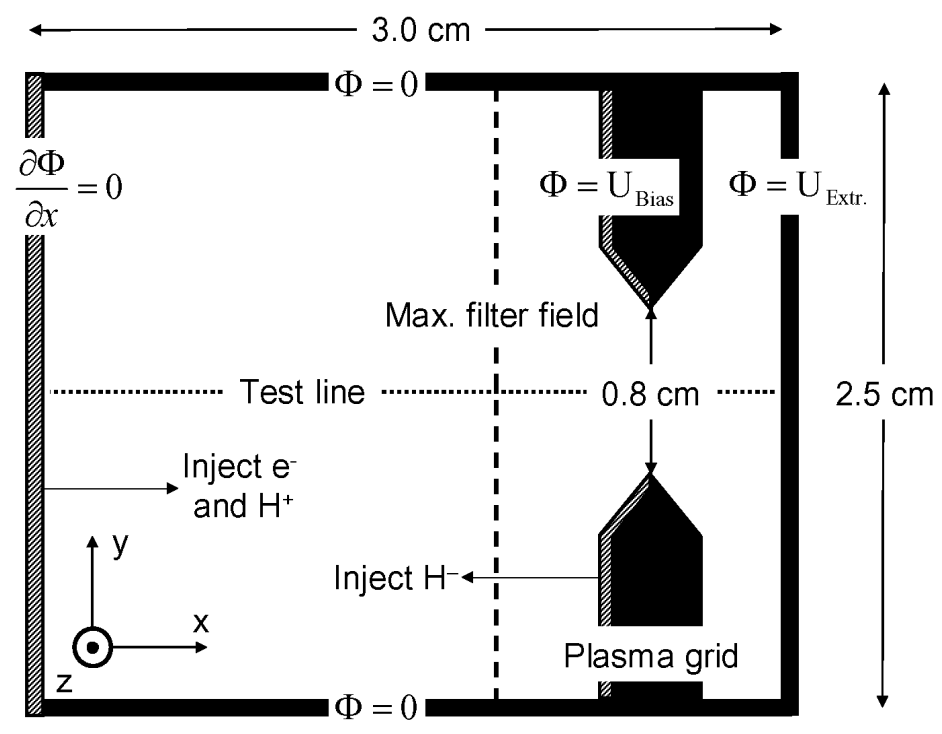

FIGURE 2. Geometry of the PIC computational domain.

1) Inject new electron and proton pseudo particles at the left boundary. For satisfying quasi neutrality equal fluxes for electrons and positive ions are used. Negative ions are injected from the surface of the plasma grid. The ratio of electron flux to negative ion flux is chosen to be 1:0.75. The velocity of the injected particles is defined using a Maxwellian flux source function [8].

2) Evaluate total charge density $\rho$ for the grid points by assigning the charge of all particles to the grid by linear interpolation.

3) Solve Poisson's equation for the electric potential $\Phi$

$$
\nabla^{2} \Phi=-\frac{\rho}{\varepsilon_{0}}
$$

using the successive overrelaxation (SOR) method [9]. The electric field is calculated by differentiating the potential.

4) Determine new velocities of the particles by solving the equation of motion:

$$
\mathbf{F}=q \cdot(\mathbf{E}+\mathbf{v} \times \mathbf{B})
$$

where $q$ and $\mathbf{v}$ are the charge and velocity of a particle as well as $\mathbf{E}$ and $\mathbf{B}$ the electric and magnetic field. A leap-frog integrator is used [6]. Thus position and velocity are shifted by half a time step which implies time-reversal invariance and hence conservation of energy. Rotation of the velocity vector due to the magnetic field is calculated by the Boris scheme [6]. A simplified 
shape which represents a kind of parabolic profile is used for the magnetic field. Just the component in y direction is incorporated:

$$
B_{y}=B_{\max } \cdot \exp \left(-\frac{(0.018-x)^{2}}{3 \cdot 10^{-4}}\right)
$$

where $\mathrm{B}_{\max }$ is the maximum field which is a parameter of the calculations and the position $\mathrm{x}$ is given in meters.

5) Calculate new positions for all pseudo particles. To conserve the total influx per time step electrons and positive ions which hit the left boundary are restarted from there with a new velocity [10]. Particles which hit one of the other walls or the electrode are removed from the simulation.

These steps are repeated until the system is in equilibrium, i.e. the number of pseudo particles and the potential in the entire simulation domain is constant.

\section{Test Particle Code}

The $3 \mathrm{~d}-3 \mathrm{v}$ test particle code calculates the extraction probability of negative ions which are produced on the surface of the plasma grid. In principle the code is similar to the model described in [11]. The computational domain consists of the volume above the extraction area of the ion source. The geometry of 73 holes (which represent half the extraction system) and their chamfered edges is included. The area around a hole in the middle of the extraction system is chosen as starting area for the test particles. In contrast to the PIC code particles are treated consecutively. Negative ions are started with an energy which represents the acceleration by the sheath voltage. The initial direction of the ions is perpendicular to the surface, broadened according to Lambert's cosine law. The trajectories of the ions are calculated numerically solving the equation of motion using the Runge Kutta method. The electric field in the plasma volume is set to zero since local variations of the electrostatic potential should be small. The magnetic field is a superposition of the filter field and the electron suppression field produced by the permanent magnets in the extraction grid. While in the simulated domain the filter field is almost constant the suppression field follows a more complex shape $\left(\mathrm{B}_{\mathrm{x}}, \mathrm{B}_{\mathrm{z}}<20 \mathrm{mT}\right)$ which has been calculated separately with a $2 \mathrm{~d}$ finite elements code.

Elastic and inelastic collisions of negative ions are calculated by the Monte Carlo method [12]. For each time step $\Delta t$ of the simulation one elastic Coulomb collision with $\mathrm{H}^{+}$ions is considered by applying a binary collision model [13]. The probability $P$ for an inelastic collision is estimated using the path length estimator algorithm [12]:

$$
P=1-\exp \left(-\sum_{j} v_{j} \cdot \Delta t\right)
$$


where $v_{j}$ is the collision frequency of the following inelastic collisions:
- Electron stripping:
$\mathrm{H}^{-}+\mathrm{e} \rightarrow \mathrm{H}+2 \mathrm{e}$
- Mutual neutralization with $\mathrm{H}^{+}: \mathrm{H}^{-}+\mathrm{H}^{+} \rightarrow 2 \mathrm{H}$
- Mutual neutralization with $\mathrm{Cs}^{+}: \mathrm{H}^{-}+\mathrm{Cs}^{+} \rightarrow \mathrm{H}+\mathrm{Cs}$
- Charge exchange:
$\mathrm{H}^{-}+\mathrm{H} \rightarrow \mathrm{H}+\mathrm{H}^{-}$

If an inelastic collision occurs within a time step, one of the four processes is chosen by a random draw [12]. Calculation of the collision frequencies requires knowledge of background particle densities. For these densities experimental results from [17, 18] are used. It is assumed that the densities are uniform over the computational domain. The values are given in table 1 .

TABLE 1. Background particle densities for the test particle code

\begin{tabular}{lc}
\hline Particle species & Density $\left[\mathbf{m}^{-3}\right]$ \\
\hline $\mathrm{e}$ & $5 \cdot 10^{17}$ \\
$\mathrm{H}$ & $10^{19}$ \\
$\mathrm{H}^{+}$ & $5 \cdot 10^{17}$ \\
$\mathrm{Cs}^{+}$ & $10^{16}$ \\
\hline
\end{tabular}

When an ion is either destroyed by a collision, hits the surface or the emission surface calculation of the trajectory is stopped and the next ion is treated.

\section{SIMULATION RESULTS AND DISCUSSION}

\section{Particle-In-Cell Code}

Electrons and protons injected at the left side of the simulation domain expand in an ambipolar manner with the ion sound velocity $\mathrm{v}_{\mathrm{c}}$. The temperatures used in the simulation $\left(\mathrm{T}_{\mathrm{e}}=2 \mathrm{eV}, \mathrm{T}_{\mathrm{H}^{+}}=0.8 \mathrm{eV}\right)$ yield a $\mathrm{v}_{\mathrm{c}}$ of approximately $2 \cdot 10^{4} \mathrm{~m} / \mathrm{s}$. Thus the plasma should hit the plasma grid after roughly 26000 time steps of $10^{-10}$ seconds. In fact the number of pseudo particles and the potential inside the simulation domain for a calculation without magnetic filter field and biasing come to a steady state after approximately 30000 time steps. When considering the filter field it takes a much longer time until equilibrium is reached.

Calculations were performed for maximum filter strengths of $0 \mathrm{mT}$ (no filter field), 1 $\mathrm{mT}, 1.5 \mathrm{mT}$ and $2 \mathrm{mT}$ respectively. The filter acts upon all particles in the simulation. Due to the huge differences in mass of the particle species $\left(\mathrm{m}_{\mathrm{H}^{+}}: \mathrm{m}_{\mathrm{H}^{-}}: \mathrm{m}_{\mathrm{e}}=1: 1: 5.48 \cdot 10^{-4}\right)$ just electrons can get fully magnetized while the trajectories of protons and negative ions are almost not disturbed. Figure 3 shows profiles of all three particle species along the test line for $1 \mathrm{mT}$. It can be seen that quasi neutrality is fulfilled in alomost the complete region in front of the emission surface: For the region in front of the filter the charge of the positive ions is compensated by the electrons while behind the filter $\mathrm{H}^{+}$and $\mathrm{H}^{-}$compensate each other. As described in [7] the flux of positive ions onto 


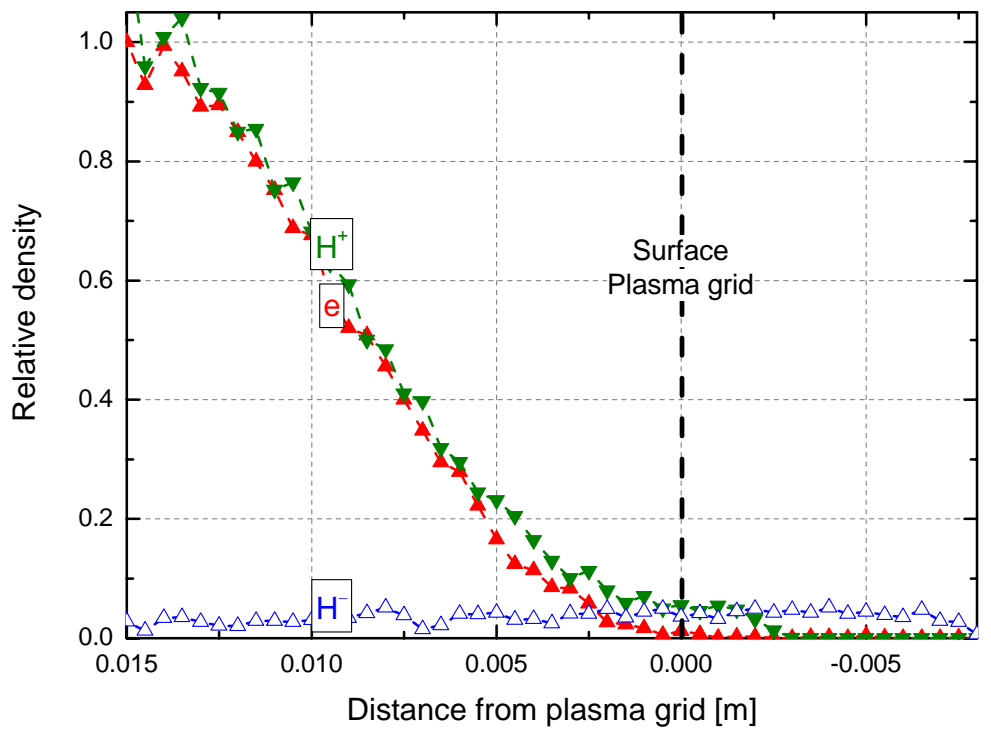

FIGURE 3. Relative particle densities along the test line for the filter field strength $1 \mathrm{mT}$.

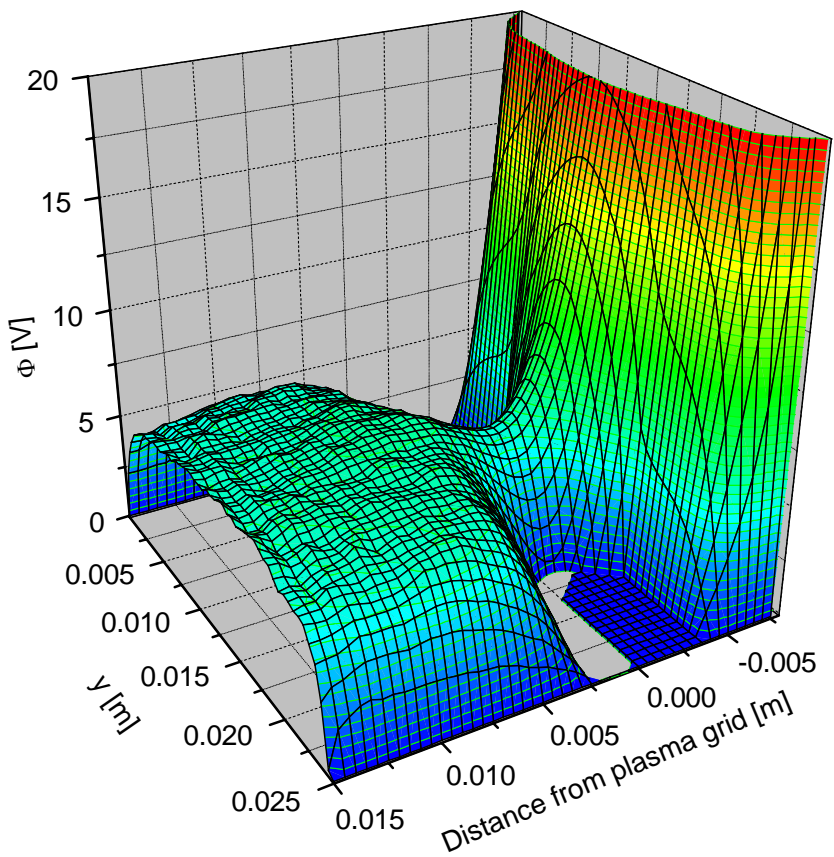

FIGURE 4. Potential for the filter field strength $1 \mathrm{mT}$

the filter is divided into two flows, the reflected back flow and the forward flow towards the plasma grid. Since quasi neutrality is fulfilled in almost the complete region in front of the plasma grid the plasma potential shows a flat shape in this region. Figure 4 shows the potential distribution for $1 \mathrm{mT}$. 


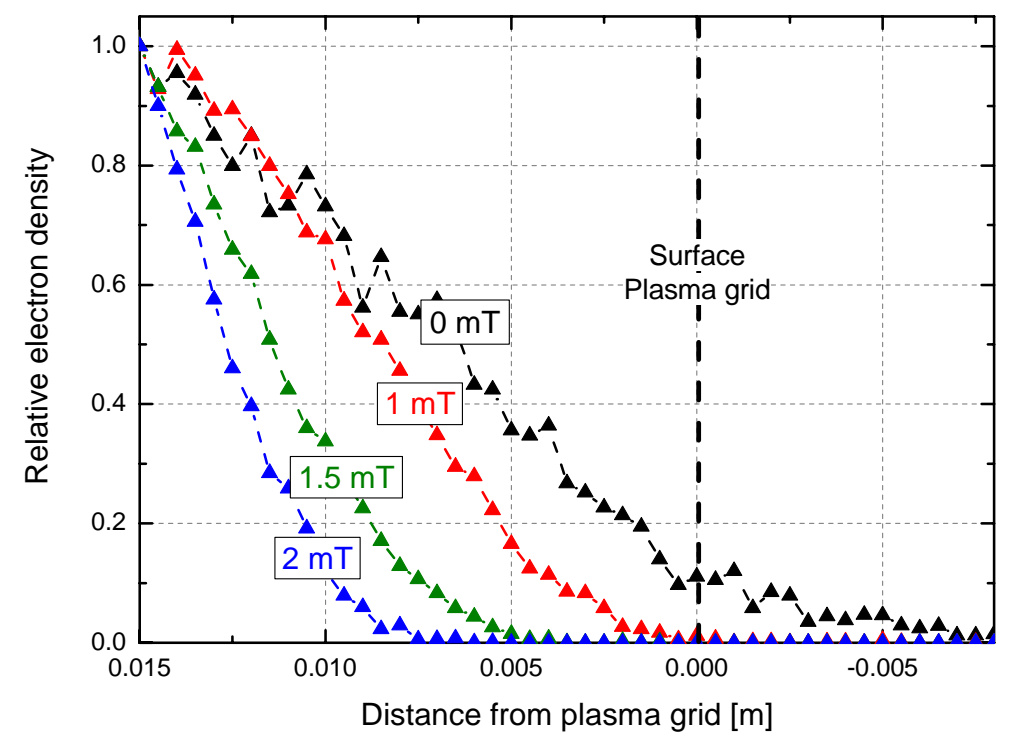

FIGURE 5. Relative electron density along the test line for different filer field strengths

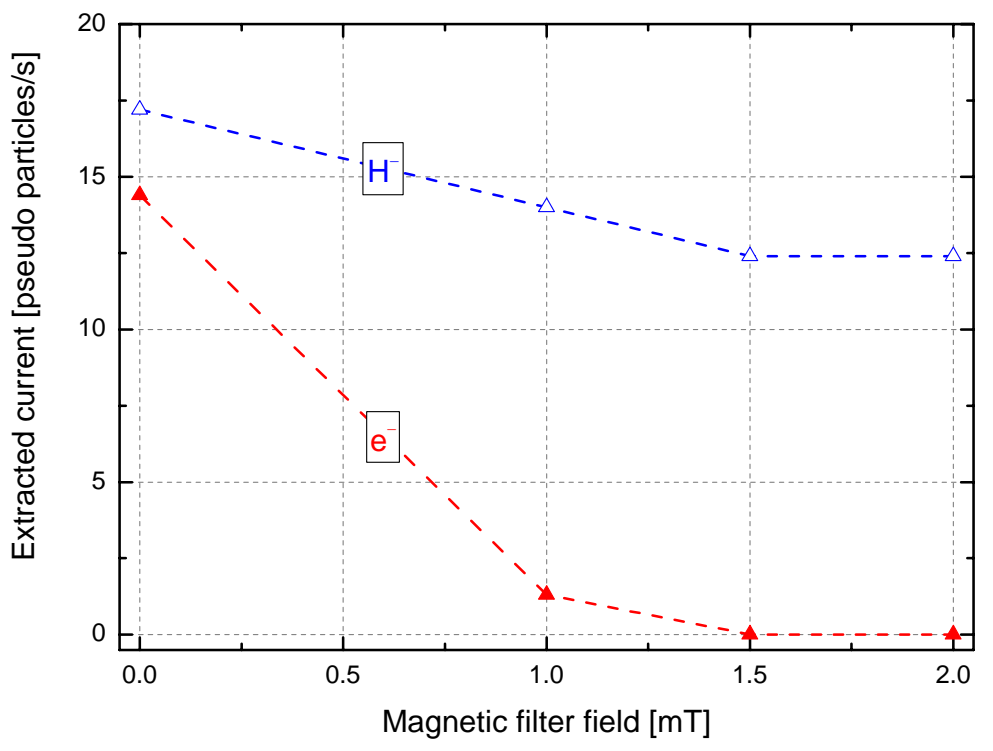

FIGURE 6. Extracted electron and ion current for different filter field strengths.

Since the Larmor radius depends on the component of the particle velocity perpendicular to the field and since the velocity of the electrons is not monoenergetic but follows a maxwellian distribution there is no threshold field strength for a complete magnetization. In fact for a given magnetic field a fraction of electrons is able to traverse the filter. This fraction falls off with increasing field strength. In Fig 5 and Fig. 6 the results of this effect can be seen. Figure 5 shows profiles of the relative 
electron density along the test line while Fig. 6 is a diagram of the extracted electron and negative ion current as a function of the field strength. The electron current is completely suppressed for filter strengths above $1 \mathrm{mT}$. In contrast the ion current does not react directly on the filter strength. The decrease shown in Fig. 6 is caused by the fact that for higher magnetic fields more negative ions are needed to preserve quasi neutrality, compensating the missing electrons. Thus increasing the filter strength increases the average mass of the negative particles present in the system. Therefore the average plasma potential decreases; from $11 \mathrm{~V}(0 \mathrm{mT})$ to approximately $6 \mathrm{~V}(2$ $\mathrm{mT})$. This is in accordance with the experiment where the plasma potential decreases with increased density of the negative ions. When implementing Cs ions in the code this effect will become more pronounced since these (positive) ions are much heavier than all particles currently regarded by the simulation and may have an important influence on the plasma potential.

In the present simulation electrons are not able to traverse a magnetic field stronger than $1.5 \mathrm{mT}$. That does not agree with reality where the peak value of the filter is around $7 \mathrm{mT}$. In such strong magnetic fields diffusion due to collisions with ions and neutral particles is the dominant process for the movement of the electrons. For this reason diffusion will be included in the next version of the model.

Several calculations were performed with modified bias voltage applied to the plasma grid. The values chosen for the bias voltage form an interval around the plasma potential $\left(U_{\text {Bias }}=5 \mathrm{~V}, 10 \mathrm{~V}, 15 \mathrm{~V}\right)$. The increase of the bias voltage leads to a small decrease of the sheath potential at the plasma grid, i.e. the acceleration voltage for the created negative ions decreases. But the vast majority of the bias voltage is transferred to a shift of the plasma potential. The reason for this effect is that the unbiased surface is almost as large as the biased electrode. Thus shifting the plasma potential by the bias potential has no large effect on the particle fluxes to the walls. This is in contrast to experimental results where the plasma potential stays almost constant when biasing the plasma grid. In the experiments the unbiased surface is much larger than the surface of the plasma grid. In previous campaigns an additional unbiased surface - the so called bias plate - was attached in front of the plasma grid [2].

The calculated electron and ion current does not vary when biasing the grid. But the decrease of the sheath potential causes changed velocities of the negative hydrogen ions. This has an effect on collision rates which can not be investigated with the PIC code at present. Therefore the test particle code was used for the following examinations.

\section{Test Particle Code}

In the test particle code the ions created at the plasma grid are started with an energy which represents acceleration by the sheath potential. In Fig. 7 typical trajectories of extracted ions (calculated for a starting energy of $1 \mathrm{eV}$ and a filter strength of $7 \mathrm{mT}$ ) are shown. The basic shape of the trajectories is mainly determined by the magnetic fields. The circular path caused by the filter field is twisted by the field of the permanent magnets in the extraction grid which is perpendicular to the filter field. The direction of trajectories may be altered abruptly by charge exchange. 


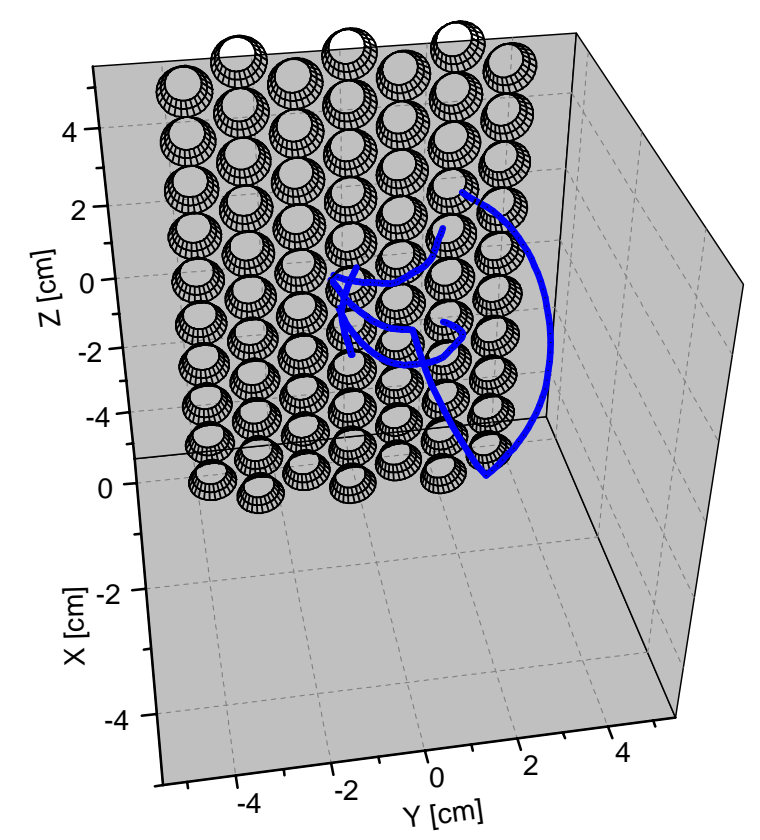

FIGURE 7. Ion trajectories calculated by the test particle code for the starting energy of $1 \mathrm{eV}$ and the filter strength $7 \mathrm{mT}$.

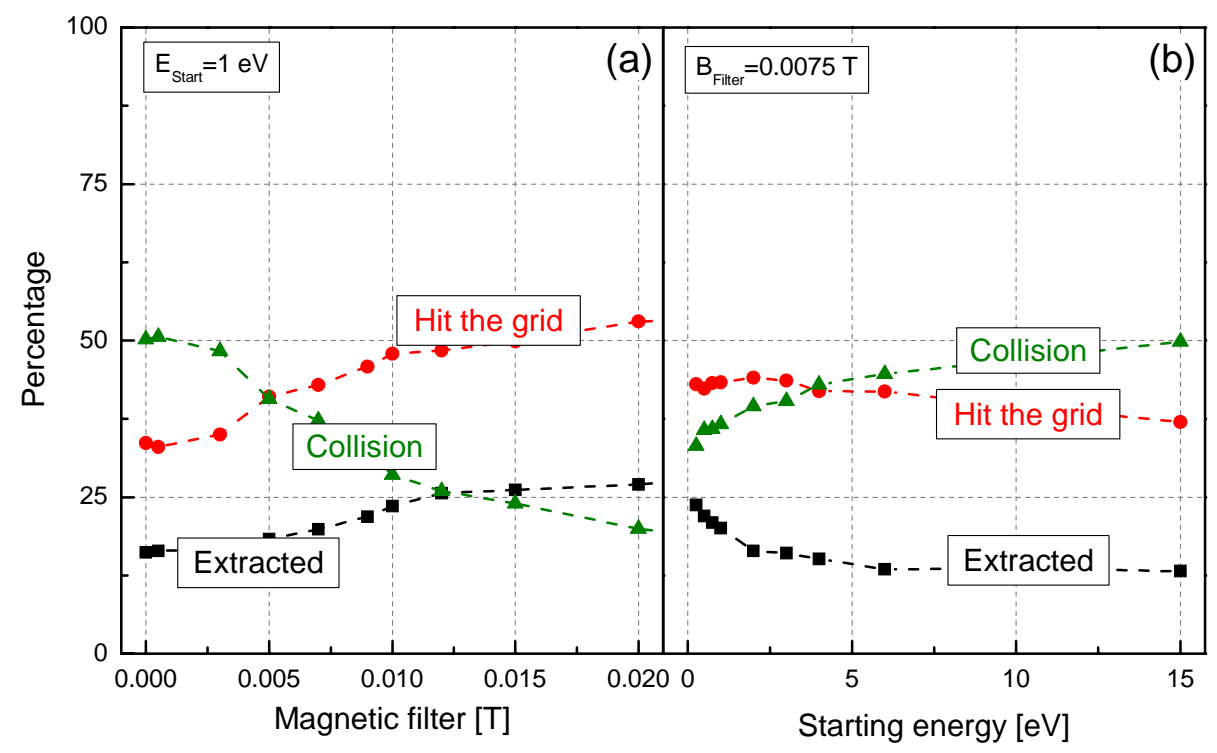

FIGURE 8. Statistics of the test particles for a variation of the magnetic filter field (a) and a variation of the starting energy (b). 
For the present plasma parameters this occurs rather frequently; the average number of charge exchange processes per trajectory is larger than one.

The code calculates the probability for the ions to be extracted, to be destroyed by a collision or to hit the plasma grid. Since there are no other possibilities the sum of these three probabilities is equal to 100 percent. Ensembles of $10^{4}$ test particles were used for the calculations.

The dependence of the probabilities on the variation of two different parameters is investigated: first of all the filter strength was varied from $0 \mathrm{mT}$ (no filter field) up to $20 \mathrm{mT}$ using a constant starting energy of $1 \mathrm{eV}$. In a second step the filter field was fixed at $7 \mathrm{mT}$ while the starting energy of the ions was varied from $0.2 \mathrm{eV}$ up to 15 eV. Results of both series are shown in Fig. $8 \mathrm{a}$ and $\mathrm{b}$ respectively.

In both series a minimum value of around 14 percent for the extraction probability is present. The reason for this large minimum is the chamfered edge of the holes. Since the particles are started perpendicular to the surface, broadened according to Lambert's cosine law the statistics always contains some particles which directly hit the emission surface, independent of the choice of the field strength or starting energy. This is in accordance with the experimental results where higher negative ion currents were extracted with chamfered edges [2].

The probability for the destruction of an ion during a single time step depends on the collision frequency. For Coulomb collisions the collision frequency is proportional to the inverse third power of the relative particle velocity while for inelastic collisions it is proportional to the density of the background particles times the rate constant. The rate constant is the product of the (energy dependent) reaction cross section with the relative particle velocity.

Changing the strength of the magnetic filter field with constant starting energy does not influence the velocity of the particles and hence does not alter the destruction probability for a single time step. However, an increased filter field directly results in a smaller radius of the trajectory and thus a smaller time until the ion hits the plasma grid or an emission surface. Since the total probability of destruction by a collision is proportional to the flight time it is also decreased. This effect can clearly be seen in Fig. 8 a.

In contrast to that changing the starting energy does result in different velocities of the particles and changed collision frequencies. As can be seen in Fig. 8 b the extraction probability decreases with increasing starting energy and reaches the minimum value for energies larger than $5 \mathrm{eV}$. This indicates that the extracted $\mathrm{H}^{-}$ current is very sensitive on the potentials inside the ion source and is in consistency with experimental results where biasing the plasma grid has a noticeable effect on the negative ion current as well as on the electron current. In the experiment a maximum extracted negative ion current is achieved when the plasma grid is biased with a potential comparable to the plasma potential, i.e. the sheath potential is minimized.

\section{CONCLUSIONS}

The influence of a magnetic filter field and biasing on the plasma and on single negative ions was studied using a PIC code and a test particle code respectively. 
Electrons flowing towards the plasma grid are magnetized and reflected into the plasma volume. Without taking into account diffusion a magnetic field of $1.5 \mathrm{mT}$ is adequate to suppress the electron flux and therefore the co-extracted electron current completely. Due to their larger mass positive and negative ions are not magnetized. Towards the grid the quasi neutrality is established by equal densities of negative and positive ions. The magnetic filter does change the average mass of the charge carriers present in the extraction region and thus influences the plasma potential and the sheath potential present at the plasma grid. A similar effect is observed when biasing the plasma grid but could be demonstrated by the PIC code just partly due to a much smaller unbiased reference surface compared to the experiment.

The extraction probability of the negative ions is sensitive on the sheath potential and the magnetic fields in the region above the plasma grid. A maximum extraction probability is reached for filter field strengths above $10 \mathrm{mT}$ and a sheath potential below $2.5 \mathrm{~V}$. Experimentally these conditions are difficult to achieve since for example changing the magnetic field also changes the sheath voltage as has been shown by the PIC code.

Although the calculations of the PIC code were performed for particle densities and magnetic field strengths which are much lower than the experimental conditions, combining the results of both codes gives a deeper insight into the processes occurring in the plasma region above the grid. In the future the PIC code will be adapted to plasma parameters which are comparable to the experimental conditions. However, this will require a lot more computing time and a parallelization of the code may be necessary.

\section{REFERENCES}

1. ITER Technical Basis 2002, ITER EDA Documentation Series No 24 (Plant Description Document, section 2.5.1), IAEA Vienna

2. E. Speth, H. D. Falter, P. Franzen, U. Fantz et al, Nucl. Fusion 46, (2006), 220

3. R. K. Janev, W. D. Langer, J. K. Evans and D. E. Post, "Elementary Processes in Hydrogen-Helium Plasmas", Springer, Berlin, 1987

4. W. G. Graham, "Properties of Alkali Metals Adsorbed onto Metal Surfaces" in Proc. $10^{\text {th }}$ Symp. on Fus. Tech., Brookhaven National Laboratory, Upton, 1980

5. M. Bacal, J. Bruneteau and P. Devynck, Rev. Sci. Instrum. 59, (1988), 2152

6. C. K. Birdsall, "Plasma Physics via Computer Simulation", Institute of Physics Publishing, Bristol, 1991

7. T. Sakurabayashi, A. Hatayama and M. Bacal, J. Appl. Phys. 95, (2004), 3937

8. R. J. Procassini, C. K. Birdsall and E. C. Morse, Phys. Fluids B 2, (1990), 3191

9. R. W. Hockney and J.W. Eastwood, "Plasma Simulation Using Particles", Taylor \& Francis, Abingdon, 1988

10. G. Gozadinos, D. Vender and M. M. Turner, J. Comp. Phys. 172, (2001), 348

11. O. Fukumasa and R. Nishida, Nucl. Fusion 46, (2006), 275

12. C. K. Birdsall, IEEE Trans. on Plasma Sci. 19, 1991, 65

13. S. Ma, R. D. Sydora and J. M. Dawson, Comp. Phys. Comm. 77, (1993), 190

14. M. J. J. Eerden, M. C. M. van de Sanden, D. K. Otorbaev and D. C. Schram, Phys. Rev. A 51, (1994), 3362

15. R. K. Janev and Z. M. Radulović, Phys. Rev. A 17, (1978), 889

16. M. A. Huels, R. L. Champion, L. D. Doverspike and Yicheng Wang, Phys. Rev. A 41 (1990), 4809

17. P. Franzen, "Status of the IPP RF negative Ion Source Development for the ITER NBI System" in Proc. $14^{\text {th }}$ Symp. on Fus. Tech., 2006

18. U. Fantz and D. Wünderlich, New J. Phys, submitted 